\title{
RIPPLES IN THE FABRIC OF SPACE-TIME
}

\author{
by Jörg Frauendiener
}

\author{
(with three text-figures and four plates)
}

\begin{abstract}
Frauendiener, J. 2016 (31:viii): Ripples in the fabric of space-time. Papers and Proceedings of the Royal Society of Tasmania 150(1): 9-14. https://doi.org/10.26749/rstpp.150.1.9 ISSN 0080-4703. Department of Mathematics and Statistics, University of Otago, P.O. Box 56, Dunedin 9054, New Zealand. Email: joergf@maths.otago.ac.nz
\end{abstract}

\begin{abstract}
Einstein's general theory of relativity is one of the finest achievements of the human mind. It has fundamentally changed the way we think about space and time, and how these in turn interact with matter. Based on this theory Einstein made several predictions, many of which have been verified experimentally. Among the most elusive phenomena that he predicted are gravitational waves, ripples in the fabric of space-time. They are disturbances in the space-time continuum which propagate with the speed of light. This contribution describes some of their properties, their sources and how it is intended to detect them.
\end{abstract}

Key Words: general relativity, gravitational waves, space-time, black holes, gravitational physics, Einstein's theory of gravitation.

\section{INTRODUCTION}

One hundred years ago, on 25 November 1915, Albert Einstein presented to the Prussian Academy of Sciences (Preussische Akademie der Wissenschaften) the final version of the field equations of gravitation which are in accordance with the principles of general relativity (Einstein 1915). He had been working on the problem of incorporating the gravitational force into his theory of relativity, which he published ten years earlier.

The main new ingredient into what he called the general theory of relativity is the formulation of the principle of equivalence. This is the formalisation of the experimental observation going back to Galilei that "all bodies fall at the same rate". Within Newtonian mechanics we can see the implications of this by writing down Newton's second law

$$
\mathbf{F}=m_{i} \mathbf{a}
$$

for a body with mass, $m_{i}$ acted upon by a force, $\mathbf{F}$. Here, we use the notation $m_{i}$ to indicate that it is the inertia of the mass which resists the attempts of the force to accelerate the body: the larger the inertial mass, $m_{i}$, the smaller the acceleration, $\mathbf{a}$. If the force is the gravitational pull exerted on a small body, $S$, by another body, $B$, such as the Earth, then

$$
\mathbf{F}=m_{g} \mathbf{g} .
$$

Here, $\mathbf{g}$ is the gravitational acceleration created by the pulling body, $B$, in case of the Earth the magnitude of this vector is $9.81 \mathrm{~m} / \mathrm{s}^{2}$ at the Earth's surface, and we use $m_{g}$ to indicate that we use the gravitational mass, that property of a body that responds to gravitational pull. Putting the two equations together we find

$$
m_{i} \mathbf{a}=m_{g} \mathbf{g} .
$$

The experimental fact that all bodies fall at the same rate, i.e., that they all respond in the same way to a gravitational pull, $\mathbf{a}=\mathbf{g}$, can be realised only if the equality $m_{i}=m_{g}$ holds. Therefore, there cannot be any difference between the inertial and the gravitational properties of a mass irrespective of its material or its interior constituents. Inertial and gravitational mass are equivalent. This is the (weak) principle of equivalence. It is the foundation on which the general theory of relativity is built upon. It is therefore essential that it is continuously checked experimentally with as many different and more accurate experiments as possible. In laboratory experiments the equivalence of inertial and gravitational mass could be checked with an accuracy of $10^{-14}$ (Baeßler et al. 1999). In 2016 a satellite experiment called MICROSCOPE (Touboul et al. 2012) will test the weak equivalence principle with a projected accuracy of $10^{-17}$.

When the principle of equivalence is valid, i.e., when all bodies respond in the same way to the gravitational pull of a massive object, then it makes sense to attribute the cause of the resulting motion of a body to the space and time continuum in which the motion takes place. This implies that space and time acquire geometric properties which determine the motion of bodies under the influence of gravity.

However, this also means that the geometry of space and time is influenced by the presence of masses such as our Sun or even larger massive objects such as neutron stars, galaxies or even black holes. More precisely, the presence of a massive object causes the space and time continuum to curve. This means that the space and time continuum can be described in a way very similar to curved surfaces such as a sphere or a torus.

The special theory of relativity had already drastically changed the way we think about space and time. The experimental fact that the speed of light is the same in all reference frames implies that time and space are intimately interwoven with each other and cannot be separated in a way which would be valid for all observers. In the words of Hermann Minkowski (address to the 80th Assembly of German Natural Scientists and Physicians on 21 September 1908):

\section{Henceforth, space by itself, and time by itself, are doomed to fade away into mere shadows, and only a kind of union of the two will preserve an independent reality. (Hilbert 1911)}

This union is called the space-time. Combining this insight from the special theory of relativity with the equivalence principle we arrive at the notion of a curved space-time which determines the motion of bodies which are subject only to 
gravitation and whose curvature in turn is influenced by the presence of matter. This emerging picture is succinctly summarised with the quotation from John A. Wheeler:

Space-time tells matter how to move; matter tells spacetime how to curve. (Wheeler \& Ford 2000, p. 235).

The interaction between the curvature of the space-time and matter is mathematically described by Einstein's Field Equation

$$
G_{a b}=\frac{8 \pi G}{c^{4}} T_{a b}
$$

where $c$ is the speed of light and $G$ is Newton's gravitational constant. It equates the Einstein tensor, $G_{a b}$, measuring certain aspects of the curvature of space-time, with the energy-momentum tensor, $T_{a b}$, characterising the dynamical energetic properties of the matter.

A solution of the Einstein equations is a space-time with a metric, a mathematical object which tells us how to measure time intervals and distances at any given point in time and space. This is the fundamental quantity which ties observers with their own private notions of time and space to all experiments that happen in space-time. In this sense, the matter contained in a space-time determines what an observer measures.

According to the Einstein equation, the Einstein tensor equals the energy-momentum tensor. But the Einstein tensor is only "half" of the curvature of the space-time. Another half is contained in the Weyl tensor, $C_{a b c d}$, a more complicated object which represents the degrees of freedom of the gravitational field. This is the object that describes the gravitational waves. The gravitational waves represented in the Weyl tensor and the matter in the Universe represented by the Einstein tensor are coupled by a system of partial differential equations which are called the Bianchi identity. It is a remarkable property and an indication of the intrinsic beauty of the theory that this system of equations is identically satisfied for any mathematical manifold with a metric. When interpreted in the context of general relativity this identity becomes the fundamental relationship between the gravitational waves and their material sources. For a more detailed presentation of the basics of the theory of general relativity in a more accessible language see Penrose (2006).

\section{THEORETICAL BACKGROUND}

The theory of general relativity (GR) views space and time as a unified entity, the space-time, which interacts with matter in a dynamical way. It changes its curvature depending on the changes in the matter. Clearly, it is a natural question as to whether the dynamics of the space-time curvature could lead to the presence of wave-like phenomena. Indeed, after a mathematical analysis of the field equations Einstein showed that they can have solutions describing small disturbances, ripples, of the space-time continuum which can propagate in a wave-like fashion with the speed of light. Consequently, Einstein (1916) predicted the existence of gravitational waves.

The exact description of such ripples in the fabric of spacetime is rather involved. The reason for the complications lies at the heart of general relativity and is an immediate consequence of the principle of relativity, another basis of the theory, which states that there are no distinguished observers (or frames of reference). This implies that the separation of a ripple (disturbance) from the undisturbed space-time cannot be done in a way that all observers would agree upon. This problem is nicely illustrated by the image given in plate 1 , which shows a view of the surface of the ocean with many "ripples" of varying sizes. Now the problem becomes obvious: where do we make the cut which separates a ripple from the undisturbed wavy ocean surface? We could say that waves with a wave-length smaller than a given size are ripples but this is an arbitrary choice and not consistent since different observers may have different notions of length (see for instance the phenomenon of Lorentz contraction in special relativity). We could also refer to the height of the water surface above the ground but this would introduce a preferred reference frame (the bottom of the ocean) into the theory which is illegal in GR.

The most successful way to obtain an objective description of gravitational waves consists of an analysis of the asymptotic behaviour. In the analogy with the ocean surface this would correspond to going to the boundary of the ocean, the beach, and watching the waves there. The analysis of the asymptotic structure of the wave-like solutions of GR goes back to the work of Bondi et al. (1962) and Sachs (1961, 1962) who proved the main result in that area, namely that gravitational waves carry away energy from a system. Newman \& Penrose $(1962,1966)$ and Penrose $(1965,2010)$ made use of the conformal properties of Einstein's theory to cast the asymptotic analysis into a geometric framework (see also Frauendiener 2004).

The current efforts in the theoretical studies of gravitational waves are largely based on computational simulations. Einstein's equation is reformulated as an initial value problem for a large system of partial differential equations which are then put on to a computer and solved under several different circumstances (Alcubierre 2012, Baumgarte \& Shapiro 2003, Choptuik et al. 2015). The goal is to determine the characteristic properties of the gravitational radiation that is emitted by different astrophysical systems. This will help to identify the sources of gravitational radiation when it is detected in the future. Ultimately, the gravitational waves will be used to obtain information about the sources and thereby will help us to understand the structure of the Universe and its contents.

The effect of gravitational waves derives from the properties of the space-time; the fact that it determines what we mean by space and time. Suppose we observe two small particles which are placed in front of us. We measure their distance. Since distance measurement is determined by the metric and hence by the curvature of the space-time this means that changes in the curvature are reflected in changes in the distance between the two bodies (and in changes in time measurements as well). Therefore, when a gravitational wave, i.e., a ripple or disturbance in the curvature of the space-time, goes through, we will see a change in the distance of the two bodies. The characteristic feature of gravitational waves is that they change distances in an anisotropic way. That means that the change is different in different directions: first of all, the waves are transversal so that there is no change in the direction of propagation of the waves and, furthermore, when the distance between two bodies increases in one direction then it decreases in the perpendicular direction. This leads to the two characteristic "plus" and "cross" polarisations of gravitational radiation shown in figure 1. 


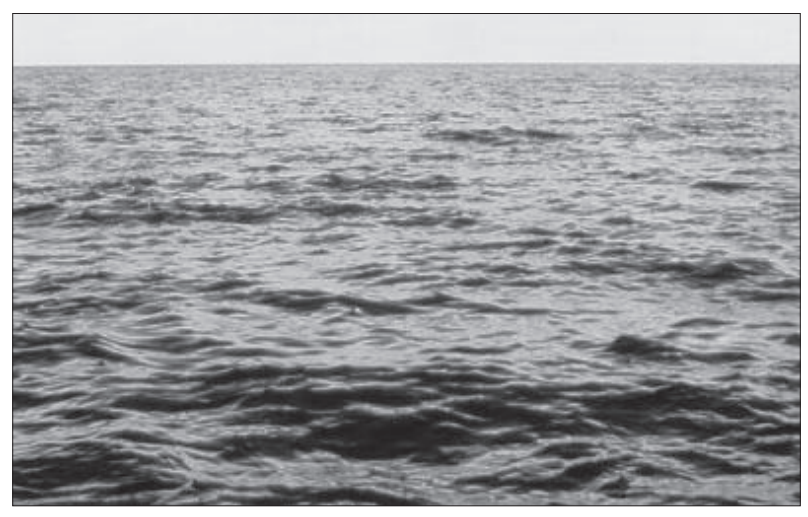

PLATE 1 - A view of the surface of the ocean. How can we distinguish between "background" and "ripples"?

\section{SOURCES OF GRAVITATIONAL WAVES}

In Maxwell's theory of electrodynamics the main source of electromagnetic radiation is an electric dipole moment which varies (in strength and orientation) over time. Thus, electromagnetic waves are generated whenever charges move; neutral particles do not emit electromagnetic waves.

In contrast, since gravitation affects all masses equally we would expect that any moving mass emits gravitational waves. Indeed, in a complete analogy to Maxwell's theory, one finds that the fundamental source of gravitational radiation in GR is a time-varying quadrupole moment. This result, the famous quadrupole formula, was derived already by Einstein (1916). It can be written (in a certain approximation) as follows

$$
\left(\frac{P}{\text { Watt }}\right) \approx 10^{-52}\left(\frac{\Omega}{H z}\right)^{6} \cdot\left(\frac{L}{m}\right)^{4} \cdot\left(\frac{M}{k g}\right)^{2}
$$

From this formula one can estimate the strength of gravitational waves. Let us assume we take a pen which has the shape of a cylinder with a length, $L=10 \mathrm{~cm}$, and a mass, $M=10$ grams. Suppose we rotate the pen around its centre of mass with a frequency, $\Omega=1 \mathrm{~Hz}$. Then the power, $P$, of the emitted gravitational radiation is of the order of $10^{-60}$ Watts. This means that one would need to wiggle some $10^{62}$ pens if one wanted to supply a 100 Watt light bulb. Therefore, in order to get an appreciable amount of gravitational wave power one needs to look at much larger systems.

The quadrupole formula tells us that the power is most sensitive to the frequency so that it is larger the more rapid the change in the quadrupole is. Also, the larger and more massive the system, the more power output there is. A large system with a changing quadrupole moment is the Earth/ Sun system. But even for that system we have a power of only 200 Watts, two light bulbs. If we consider Jupiter instead of Earth we achieve about 5300 Watts. Thus, even for systems of astronomical size one gets a comparatively small amount of power. We need to find large systems which change very rapidly. Such systems do exist. They consist of neutron stars or black holes, very compact, very massive objects which circle around each other very much like the Earth/Sun system. These binary systems are the prime sources for strong gravitational radiation. One particular
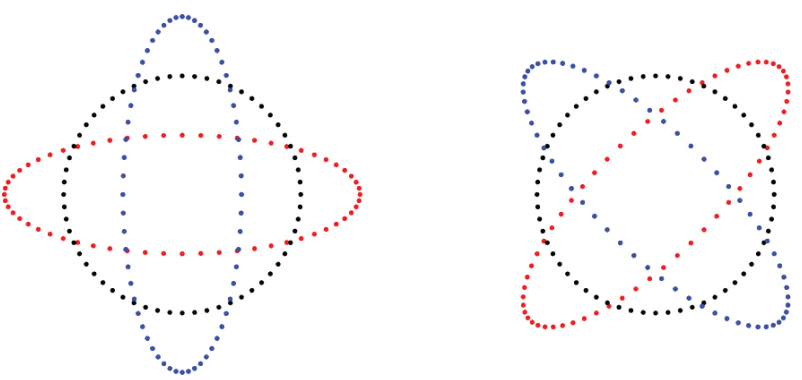

FIG. 1 - The two different polarisation states of a gravitational wave. The pictures show the effect of the "plus" (left) and "cross" (right) waves in a ring of particles (black). Over one wavelength it is distorted first into the red configuration then back to the black one and then into the blue configuration.

such system, the so-called double pulsar, was discovered in 2003 (Kramer et al. 2006). It consists of two neutron stars each with roughly the mass of the Sun which circle around each other in 144 minutes at a distance of only $850000 \mathrm{~km}$. The power emitted by this system is of the order of 1045 Watts.

While the binary systems are probably the most prominent sources for gravitational waves, there also exist others. They can be divided into four different kinds according to the signals they emit: inspiral, burst, continuous and stochastic. The first class corresponds to the late stages of a binary system which has already lost so much energy that the two partners are very close to each other and the frequency of the signal is very high. Then the partners will ultimately fall onto each other and merge. The signal is expected to be "chirp"-like.

The burst signal might come from such objects as exploding supernovae or gamma ray bursts. Due to the lack in the microphysical understanding of these systems not much can be said about the detailed structure of the signals.

The continuous signals are expected to come from almost stationary systems such as a young binary or a neutron star with an irregular non-axisymmetric shape (a mountain). These signals are expected to be quite weak and not detectable with the current generation of detectors.

The stochastic signals are the relic of the "big bang" in much the same way as the cosmic microwave background $(\mathrm{CMB})$. The difference is that the electromagnetic waves of the CMB were created at the surface of last scattering (or recombination) at about 300000 years after the "big bang", while the gravitational waves we would see were created very shortly after the "big bang". The signal will be stochastic and isotropic (i.e., noise from all directions).

\section{OBSERVATIONS}

As described in the previous section, gravitational waves are everywhere since every moving mass emits them. However, they are generally very weak. So how do we know that they exist? We have two pieces of evidence for their existence but a direct detection has remained elusive to this day.

The first piece of evidence for the existence of gravitational waves is purely theoretical and consists of the mathematical fact that Einstein's theory which has been tested and confirmed in several other contexts predicts the wave-like 


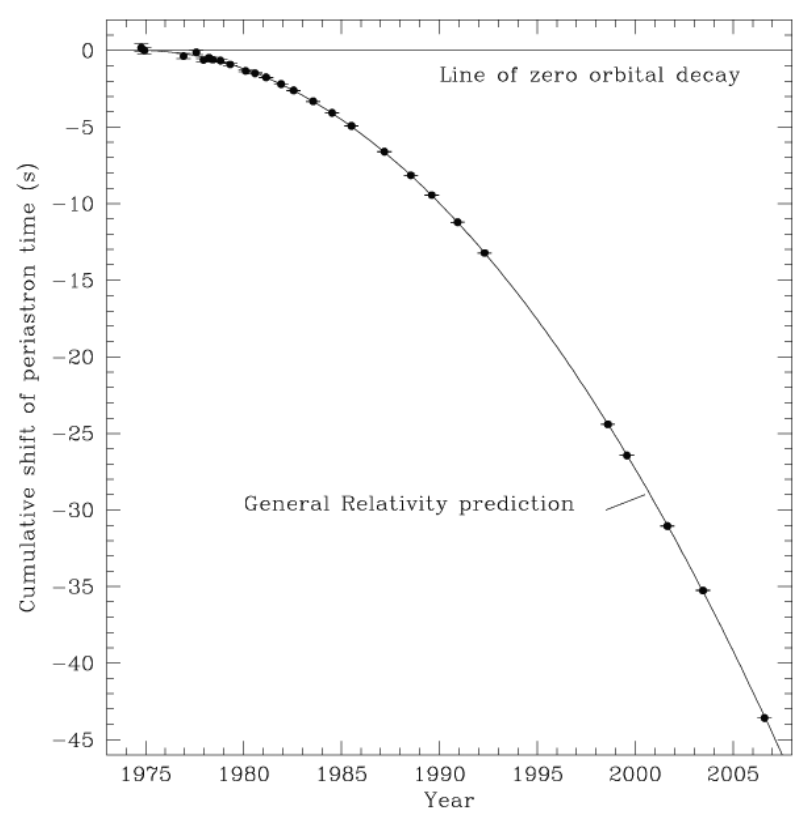

FIG. 2 - The change in the period of the binary pulsar observed and predicted by GR.

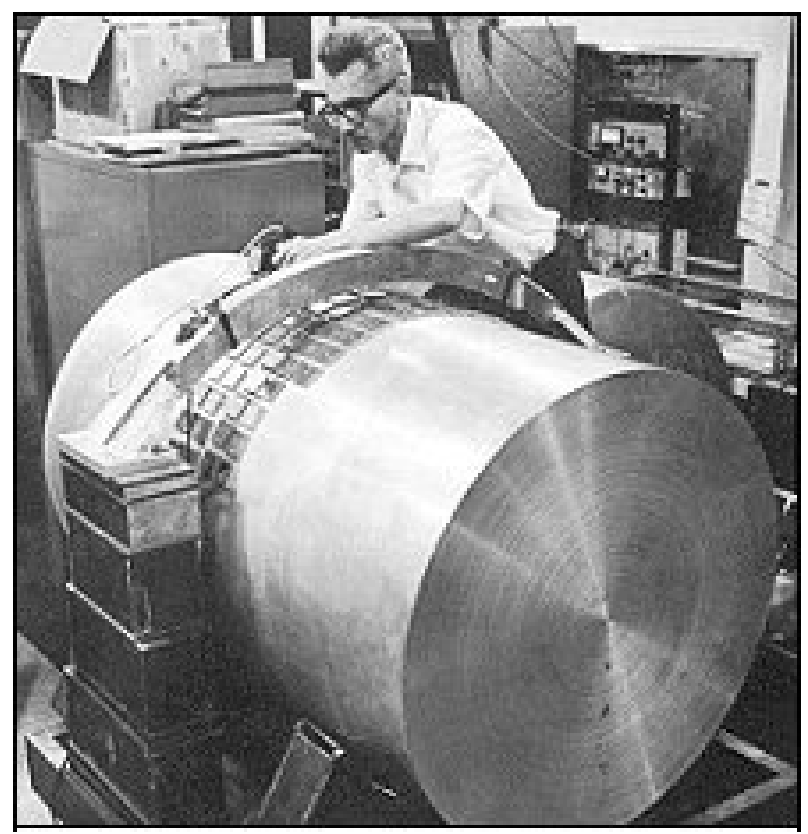

PLATE $2-\mathrm{J}$. Weber with one of his bar detectors.

solutions which carry energy. Hence, it is very likely that this aspect of the theory is correct as well.

The other piece of evidence is an indirect experimental verification. The first binary system, consisting of a pulsar and an ordinary star, was discovered in 1973 by Hulse \& Taylor (1974). It was subsequently monitored continuously and its parameters were registered over a time of more than three decades. It was found that the period of the system decreases over time. This means that the system loses energy, the two bodies come closer to each other so that they rotate faster (just like an ice skater who brings in her arms to perform a pirouette). But the energy that is lost must go somewhere and the most reasonable explanation is that the system emits gravitational waves which carry the energy away. One can estimate the amount of emitted gravitational radiation using the quadrupole formula and, based on that, one can determine the change of the period of the system from the beginning of the observation. The result is the graph presented in figure 2.

The graph shows measurements of the period of the binary pulsar between 1973 and 2007. The horizontal line at the top corresponds to the behaviour when no energy is lost. The solid line gives the behaviour if the energy loss is caused by gravitational waves according to GR and the dots are the actual measurements with their error bars. The agreement is obvious.

In the meantime, several binary systems have been found and observed with the same results. While it is quite comforting to know that these systems behave exactly as Einstein's theory predicts, there remains some uneasiness since we still do not have a direct proof of the existence of gravitational waves.

A direct detection of the gravitational waves is difficult. Not only are their possible sources very far away, the waves by themselves are very weak as we have seen. This means that the detectors must be of a very high quality in order to be able to "see" something. The detection uses the effect of gravitational waves on bodies as discussed previously: either one monitors the distance between free bodies or the force between fixed bodies that is needed in order to keep them in place when the gravitational wave grabs them.

Correspondingly, gravitational wave detectors come in two flavours, laser-interferometers and bar detectors. Historically, the bar detectors were first. The first attempt of detection was made with a bar detector by Joseph Weber (pl. 2) in the 1960s. He used two aluminium cylinders, each about $1.5 \mathrm{~m}$ long and $0.5 \mathrm{~m}$ thick. When a gravitational wave comes through them it rattles the atoms in the aluminium and causes them to vibrate at a characteristic frequency that is detected using piezo-electric transducers. In 1968, he claimed that he had detected gravitational waves but this was soon disputed and even today we have not seen these waves. Bar detectors are still in use, see for instance MiniGRAIL (De Waard et al. 2003) in Leiden (pl. 3).

The disadvantage of bar detectors is that they are sensible only for a very small frequency window dictated by the geometry of the bar. Therefore, most gravitational wave detectors today are based on the laser-interferometric design. The detectors operate like a Michelson interferometer monitoring the length of two arms perpendicular to each other using laser beams which are reflected at the end of the arms by a highly reflecting mirror and brought to interference back at the intersection of the two arms (see fig. 3).

When a gravitational wave goes through it changes the arm-lengths in opposite ways as described in the section on sources of gravitational waves and the relative change is detected. The size of this change is estimated to be at the order of $10^{-22}$, corresponding to the inverse ratio of the distance between Sun and Saturn to the diameter of a hydrogen atom. This is a minute change in length which implies that the equipment must be of the utmost quality.

There are currently four interferometers in operation under the umbrella of the LIGO Scientific Cooperation (LSC): the two LIGO detectors in Livingstone, Louisiana, and Hancock, Washington, with an arm-length of $4 \mathrm{~km}$ (see pl. 4); GEO600 in Hannover, Germany, with $600 \mathrm{~m}$ arm-length and VIRGO in Pisa, Italy, with an arm-length of $3 \mathrm{~km}$. These detectors are operating simultaneously in order to catch coincidences. They have recently been upgraded to a more advanced technology and will soon 
start to take data at a much higher sensitivity. Two further detectors are planned: KAGRA in Japan and the Einstein telescope in Europe.

The ground-based detectors in use today are limited in their sensitivity at low frequencies by several sources of noise, the most important one being the seismic noise that is generated by irregularities in the environment. This is the reason why several years ago plans were developed to send a satellite-based detector in an orbit around the Sun. The idea is to have three satellites in a fixed configuration at a mutual distance of several million kilometres trailing behind the Earth and send laser beams between them in order to monitor their relative distances.

This project, called LISA (Laser-Interferometric Space Antenna), went through several ups and downs and its development has been delayed for a number of years. However, on 3 December 2015 the European Space Agency launched a precursor, the LISA Pathfinder mission, to test that two masses can fly through space and maintain their relative positions to the precision required for the operation of a gravitational wave observatory.

\section{THE FUTURE}

With the technological advances that have gone into the recent upgrade of the currently operating gravitational wave detectors the community seems to be cautiously optimistic that a direct detection of the gravitational waves will happen within the next five years. Of course, this would be a triumph for the theory of general relativity in that all of Einstein's original predictions have been verified.

However, this is not the only consequence. With the detection of gravitational waves we will have another medium at our disposal in addition to the full spectrum of electromagnetic waves. This will allow us to gain much more information about the Universe and the matter it contains than we currently have. Until now we obtain all our information about the Universe via electromagnetic radiation (light, $\mathrm{X}$-rays, gamma rays, etc.). However, as is well known, these waves are easily reflected, scattered, absorbed and reemitted. Therefore, the signals we receive contain information about all the intervening processes. There is a limit beyond which we cannot see back into the past because the electromagnetic radiation was completely scattered by the hot plasma that first formed after the "big bang". When the Universe had cooled down enough so that the first atoms could form ("recombine") from the plasma, then the photons could travel freely without being scattered. Therefore, electromagnetic radiation cannot provide us with information about the time before the recombination. However, the Universe is completely transparent to gravitational waves which will allow us to get information about times as far back as the "big bang".

Furthermore, the detailed physics of how the radiation is emitted is encoded in both channels of gravitational and electromagnetic radiation. Their combined observation will provide us with many new insights into the microphysics of the sources, such as neutron stars, supernovae and gamma ray bursts.

In summary, the successful detection of gravitational waves will open a new window to the Universe and initiate the new era of gravitational wave astronomy.

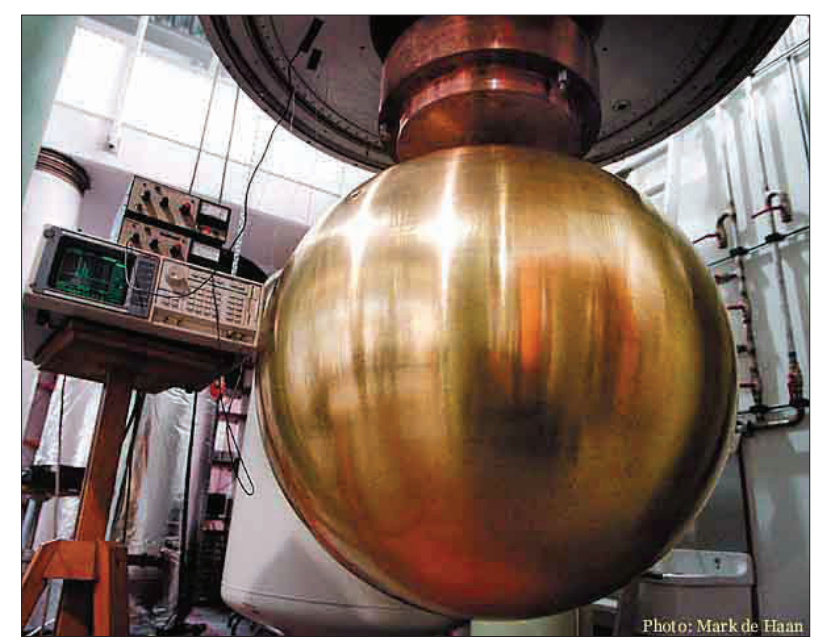

PLATE 3 - The spherical detector MiniGRAIL in Leiden.

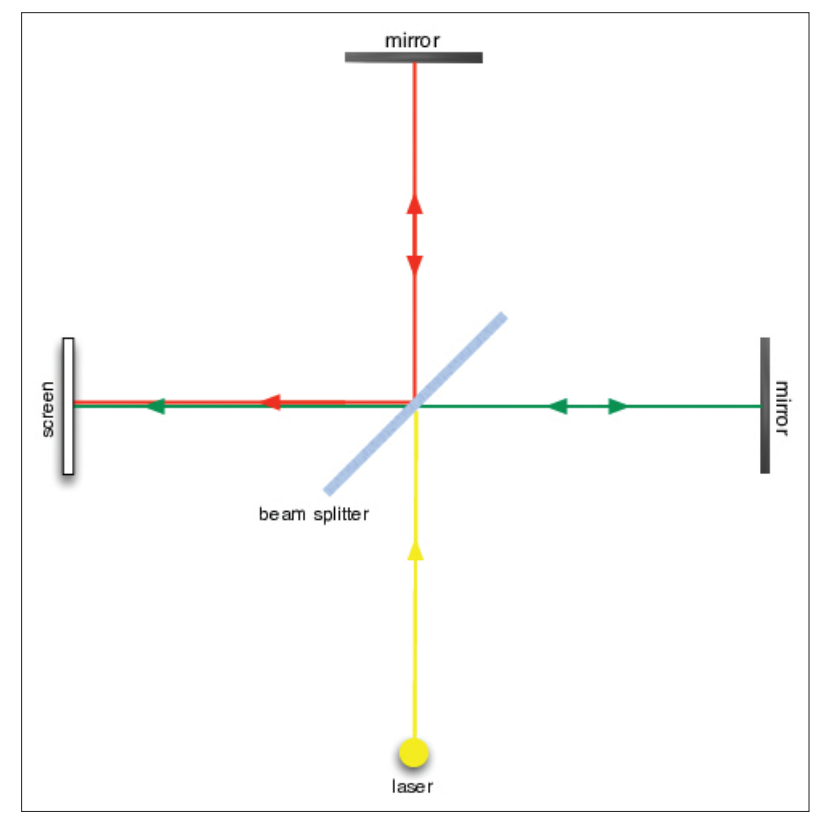

FIG. 3 - Schematic diagram of an interferometric gravitational wave detector. A laser sends light into the interferometer which is split by a splitter into two rays perpendicular to each other. After reflections at the mirrors the beams are recombined and compared.

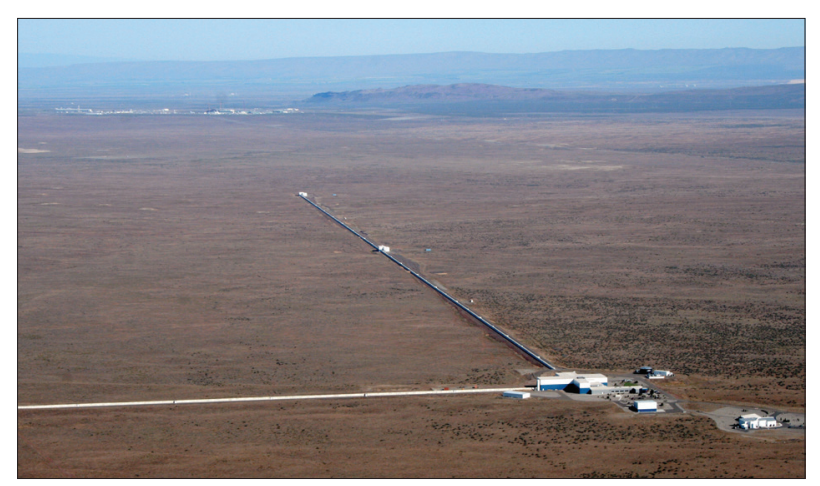

PLATE 4 - An aerial view of the LIGO site in Hancock, Washington. Note the two arms, each with a length of $4 \mathrm{~km}$. The beam splitter and the detection electronics are located in the central building. 


\section{NOTE ADDED AT REVIEW STAGE}

The future has arrived! While this article was in the reviewing process, the Ligo Scientific Collaboration (LSC) published the details of two detection events: GW150914 (Abbott et al. 2016a) and GW151226 (Abbott et al. 2016b) were both received by the two LIGO detectors in the USA and were both caused by black holes merging into a single one.

The earlier signal from 14 September 2015 was emitted by two black holes with masses of around 36 and 29 solar masses. They came together within 0.2 seconds having circled around each other with a frequency of up to $250 \mathrm{~Hz}$. They settled into a single black hole with a mass of 62 suns, having emitted gravitational waves with a total energy of the equivalent of three solar masses. To put this into perspective: the most energetic man-made event was the so-called "Tsar Bomba", a soviet hydrogen bomb with 50 mega-tons TNT, which detonated in 1961. This energy output corresponds to a rest-mass equivalent of just $2 \mathrm{~kg}$.

The second event was much smaller. It was recorded on Boxing Day 2015. Again, two black holes collided and merged within 1 second. Their masses were 14.2 and 7.5 solar masses, respectively.

These detections confirmed Einstein's theory twice: they showed that gravitational waves are real, thereby putting an end to a one hundred-year-old uncertainty. But they also confirmed that black holes exist because there are no other currently known massive systems that could have created the first event GW150914. This shows that the Kerr-Newman family of solutions to the Einstein equations is of important physical relevance.

Based on the two detections to date it is estimated that there will be on the order of 1000 detections per year once the detectors have reached their final sensitivity. These are certainly exciting times to be a researcher in gravitational wave physics.

\section{ACKNOWLEDGEMENTS}

The author is very grateful to the organisers of the Biennial meeting of Australian and New Zealand Alexander von Humboldt Fellows in Hobart where this paper was accepted for oral presentation.

\section{REFERENCES}

Abbott, B.P., together with 1011 other authors, 2016a: Observation of gravitational waves from a binary black hole merger. Physical Review Letters 116: 061102.

Abbott, B.P., together with 969 other authors, 2016b: GW151226: Observation of gravitational waves from a 22-solar-mass binary black hole coalescence. Physical Review Letters 116: 241103.

Alcubierre, M. 2012: Introduction to 3+1 Numerical Relativity. Oxford University Press, Oxford: 444 pp.

Baeßler, S., Heckel, B., Adelberger, E., Gundlach, C., Schmidt, U. \& Swanson, H. 1999: Improved test of the Equivalence
Principle for Gravitational Self-Energy. Physical Review Letters 83: 3585-3588.

Baumgarte, T.W. \& Shapiro, S.L. 2003: Numerical relativity and compact binaries. Physics Reports 376: 41-131.

Bondi, H., Van Der Burg, M.G.J. \& Metzner, A.W.K. 1962 Gravitational waves in general relativity. VII. Waves from axi-symmetric isolated systems. Proceedings of the Royal Society London A 269: 21-52.

Choptuik, M.W., Lehner, L. \& Pretorius, F. 2015: Probing strong field gravity through numerical simulations, In A. Ashtekar, B. Berger, J. Isenberg \& M.A.H. MacCallum (eds.): General Relativity and Gravitation: A Centennial Perspective. Cambridge University Press, Cambridge, 2015: 361-411.

De Waard, A., Gottardi, L., van Houwelingen, J., Shumack, A. \& Frossati, G. 2003: MiniGRAIL, the first spherical detector. Classical and Quantum Gravity 20: S143-51.

Einstein, A. 1915: Die Feldgleichungen der Gravitation. Sitzungsberichte der Preussischen Akademie der Wissenschaften zu Berlin 2: 844-847.

Einstein, A. 1916: Näherungsweise Integration der Feldgleichungen der Gravitation. Sitzungsberichte der Königlich Preussischen Akademie der Wissenschaften 1: 688-691.

Frauendiener, J. 2004: Conformal infinity Living Reviews in Relativity 7 2004-1, doi: 10.12942/lrr-2004-1.

Hilbert, D. (ed.) 1911: Gesammelte Abhandlungen von Hermann Minkowski, Teubner, Leipzig and Berlin: $871 \mathrm{pp}$.

Hulse, R.A. \& Taylor, J.H. 1974: A high-sensitivity pulsar survey. Astrophysical Journal 191: L59.

Kramer, M., Stairs, I.H., Manchester, R.N., McLaughlin, M.A. Lyne, A.G., Ferdman, R.D., Burgay, M., Lorimer, D.R., Possenti, A., D’Amico, N., Sarkissian, .J, Joshi, B.C., Freire, P.C.C. \& Camilo, F. 2006: Strong field tests of gravity with the double pulsar. Annalen der Physik 15: 34-42.

Newman, E.T. \& Penrose, R. 1962: An approach to gravitational radiation by a method of spin coefficients. Journal of Mathematical Physics 3: 566-578.

Newman, E.T. \& Penrose, R. 1966: Note on the Bondi-MetznerSachs group. Journal of Mathematical Physics 7: 863-70.

Penrose, R. 1965: Zero rest-mass fields including gravitation: asymptotic behaviour. Proceedings of the Royal Society London A 284: 159-203.

Penrose, R. 2006: The Road to Reality. Random House, London: 1136 pp.

Penrose, R. 2010: Republication of: Conformal treatment of infinity. General Relativity and Gravitation 43: 901-922.

Sachs, R.K. 1961: Gravitational waves in general relativity. VI. The outgoing radiation condition. Proceedings of the Royal Society London A 264: 309-38.

Sachs, R.K. 1962: Gravitational waves in general relativity. VIII. Waves in asymptotically flat space-time. Proceedings of the Royal Society London A 270: 103-26.

Touboul, P., Metris, G., Lebat, V. \& Robert, A. 2012: The MICROSCOPE experiment, ready for the in-orbit test of the equivalence principle. Classical and Quantum Gravity 29: 184010.

Wheeler, J.A. \& Ford, K. 1998: Geons, Black Holes, And Quantum Foam: A Life in Physics. WW Norton \& Company, New York: $416 \mathrm{pp}$.

(Accepted 5 July 2016) 